\title{
A RETROSPECTIVE DESCRIPTIVE STUDY OF INCIDENCE AND OUTCOME OF RETINOPATHY OF PREMATURITY IN LEVEL-3 NICU IN A TERTIARY CARE CENTRE
}

\author{
Rachamadugu Uma Devi' ${ }^{1}$ S. Srikanth Bhatt², Gummadi Anjani ${ }^{3}$ \\ ${ }_{1}^{1}$ Associate Professor, Department of Paediatrics, Gandhi Medical College, Secunderabad. \\ ${ }^{2}$ Associate Professor, Department of Paediatrics, Gandhi Medical College, Secunderabad. \\ 3 Senior Resident, Department of Paediatrics, Gandhi Medical College, Secunderabad.
}

\section{ABSTRACT}

\section{BACKGROUND}

Retinopathy of Prematurity (ROP) is a vasoproliferative disease affecting the eyes of premature infants. ROP continues to be one of the important preventable causes of childhood blindness. The most important determinant of any ROP management program is an effective screening strategy.

\section{MATERIALS AND METHODS}

An ROP retrospective descriptive study was performed for a duration of 6 months between January 2017 and June 2017, in which babies with a gestational age of 34 weeks or less and a birth weight of $2000 \mathrm{~g}$ or less were included. Infants whose gestational age was more than 34 weeks or birth weight more than $2000 \mathrm{~g}$ were included if babies had any other comorbidities. The study was done by performing ROP screening in babies, staging the disease and documenting the risk factors. The outcome and management of those diagnosed with ROP was noted too.

\section{RESULTS}

A total of 160 babies ( 85 males and 75 females) had retinal evaluation from the fourth postnatal week and followed up periodically. Sixteen infants developed any stage ROP accounting for ten percent incidence among all the enrolled and screened babies. Stage I ROP was seen in nine infants, Stage II in three infants and Stage III in two infants. Stage IV or V ROP was detected in one case each. Male predominance was noted in the study. Respiratory distress and sepsis were noted to be the most frequent risk factors. Of all sixteen cases that developed ROP, 8 cases required laser photocoagulation accounting to 50 percent of ROP cases and five percent of the total screened infants.

\section{CONCLUSION}

Screening for ROP aids in timely diagnosis, would facilitate early interventional strategies leading to prevention of ocular morbidity and blindness. The incidence of ROP is relatively less in our setup, probably due to monitored oxygen therapy and proper screening protocols.

\section{KEYWORDS}

Prematurity, ROP, Screening, Laser Photocoagulation, Sepsis, Respiratory Distress Syndrome.

HOW TO CITE THIS ARTICLE: Devi RU, Bhatt SS, Anjani G. A retrospective descriptive study of incidence and outcome of retinopathy of prematurity in level-3 NICU in a tertiary care centre. J. Evolution Med. Dent. Sci. 2018;7(01):82-85, DOI: 10.14260/jemds/2018/19

\section{BACKGROUND}

Retinopathy of prematurity (ROP) is a vasoproliferative disease affecting the eyes of premature infants. ROP continues to be one of the important preventable causes of childhood blindness.(1-3) With the increased survival of premature infants due to improvement in neonatal care there has been an alarming increase in the incidence of ROP, especially in the developing countries.(4,5) The clinical profile of infants with ROP in developing countries like India and that in developed countries is different. Out of 26 million annual live births in India, approximately 2 million are $<2000 \mathrm{~g}$ in weight and are at risk of developing ROP. In India, the incidence of ROP is between 38 and $51.9 \%$ in low-birth weight infants. $(6,7)$

'Financial or Other Competing Interest': None.

Submission 23-10-2017, Peer Review 11-12-2017,

Acceptance 19-12-2017, Published 01-01-2018.

Corresponding Author:

Dr. S. Srikanth Bhatt,

Flat No. 403, Plot No. 29,

Annapurna Residence, Auto Sai Nagar,

Vanasthalipuram, Hyderabad-70.

E-mail: srikanthbhatt05@gmail.com

DOI: $10.14260 /$ jemds/2018/19
The aetiology of ROP is multifactorial. Low birth weight, lower gestational age, supplemental oxygen therapy and sepsis are among the major risk factors for the development of ROP. $(2,8,9)$

Retinopathy of prematurity (ROP) was originally designated as retrolental fibroplasias by Terry in 1952 who related it with premature birth.(10) Term ROP was coined by Heath in 1951.(11)

The most important determinant of any ROP management program is an effective screening strategy. As per western screening guidelines, which include infants with gestational ages of 30 weeks or $6 / 7$ weeks post birth or less (irrespective of birth weight) and birth weights of $1500 \mathrm{~g}$ or less for screening, Indian scenario seems to have been missing on few babies as per these criteria and hence need to be modified according to our scenario. Early treatment has been shown to improve a baby's chances for normal vision.

Treatment includes cryotherapy and laser therapy (photocoagulation) is used to prevent complications of advanced ROP. Surgery is done for detached retina, but may not result in good outcome.

The objective of this study was to evaluate the incidence of ROP in a tertiary care hospital, to analyse the distribution of 
risk factors for the development of ROP, the outcome of ROP and to increase awareness among the health care providers.

\section{MATERIALS AND METHODS}

The retrospective descriptive study was conducted in neonatology unit, Department of Paediatrics, Gandhi Hospital, a tertiary care medical college hospital in Secunderabad, Telangana state. The study duration was for a period of six months between January 2017 and June 2017. Inclusion criteria included gestational age of 34 weeks or less, birth weight equal to or less than 2000 gms. Presence of other factors such as respiratory distress syndrome, sepsis, multiple blood transfusions, multiple births (twins/ triplets), apneic episodes and intraventricular haemorrhage increase the risk of ROP, and hence included in the study group. The study was done by performing ROP screening in babies, staging the disease and documenting the risk factors. A detailed documentation including gestational age, birth weight, perinatal risk factors, respiratory distress, birth asphyxia, sepsis and neonatal jaundice was done.

All the enrolled infants underwent pupillary dilatation with $0.2 \%$ tropicamide and $1.25 \%$ phenylephrine eye drops, instilled every ten minutes for three applications. Examination was performed by indirect ophthalmoscopy with 20 diopters lens 30 to 40 minutes later. The first screening was done at three or four weeks postnatal depending upon the clinical status of babies. The follow-up examinations were based on the initial findings. After that they were followed until full vascularisation of the retina had reached zone 3 or until full remission of ROP, after treatment and diode laser photocoagulation of the peripheral avascular retina. Classification of ROP was done according to the International Classification of ROP (ICROP) ${ }^{(12)}$ depending on the severity as stage 1 - 5 .

\section{International Classification of Retinopathy Guidelines has classified ROP into the following stages}

- Stage I: There is a line demarcating the vascular and avascular retina.

- $\quad$ Stage II: Development of a 'ridge,' signifying increased disease activity.

- Stage III: Development of 'extraretinal' vascular and fibrous components with a propensity to bleed.

- Stage IV: Partial detachment of the retina, either involving the periphery only $(4 \mathrm{~A})$ or the centre of vision as well (4B).

- $\quad$ Stage V: Total retinal detachment.

The follow-up program was carried according to the stage as per guidelines.(7) Early treatment of high-risk ROP significantly reduced unfavourable outcomes to a clinically important degree and hence laser treatment protocol is followed according to (ETROP) Early Treatment for Retinopathy Of Prematurity Cooperative Group.(13)

Statistical method and analysis was performed using SPSS version 22. Descriptive statistics are mentioned in percentages, mean and standard deviations.

\section{RESULTS}

A total of one hundred and sixty babies were enrolled in the study group and followed up accordingly. The cases include 85 males and 75 females. The mean gestational age was 33.5 weeks (range 26 to 40 weeks) and the mean birth weight was 1540 grams ( 900 to 3800 grams).

\begin{tabular}{|c|c|}
\hline Characteristic Feature & Value \\
\hline Total no. of babies (\%) & $160(100 \%)$ \\
\hline Total no. of males (\%) & $85(53.1 \%)$ \\
\hline Total no. of females (\%) & $75(46.9 \%)$ \\
\hline Mean gestation age (weeks) & $33.5( \pm 2.6 \mathrm{SD})$ \\
\hline Mean birth weight (Grams) & $1540( \pm 370$ SD) \\
\hline \multicolumn{2}{|c|}{ Table 1. Study Group Characteristics } \\
\hline
\end{tabular}

Of the 160 babies screened 21 infants were less than 30 weeks gestational age (GA), 88 infants were between 31 - 34 weeks GA and 51 infants were more than 34 weeks GA.

Sixteen infants developed any stage ROP accounting for ten percent incidence among all the enrolled and screened babies. Stage I ROP was seen in nine infants, Stage II in three infants and Stage III in two infants. Stage IV or V ROP was detected in one case each.

\begin{tabular}{|c|c|c|}
\hline Stage & No. of Cases & Percentage \\
\hline Stage 1 & 9 & 56.25 \\
\hline Stage 2 & 3 & 18.75 \\
\hline Stage 3 & 2 & 12.5 \\
\hline Stage 4 & 1 & 6.25 \\
\hline Stage 5 & 1 & 6.25 \\
\hline Total & $\mathbf{1 6}$ & $\mathbf{1 0 0}$ \\
\hline \multicolumn{3}{|c|}{ Table 2. ROP Staging } \\
\hline
\end{tabular}

Among the infants that developed ROP, 6 infants (37.5\%) were in less than 30 weeks, 1 infant (6.25\%) was in more than 34 weeks and 9 infants (56.25\%) were in 31 - 34 weeks gestational age. The average birth weight of babies with ROP stage 1 to stage 5 is 1293 grams ( 990 grams - 1600 grams) with SD \pm 194 grams and mean GA was $31.4( \pm 2.25)$ weeks. Of the 16 cases, 13 cases were male babies accounting for $81.25 \%$ and 3 were female babies. Among the 16 infants with ROP changes, eight infants had plus disease.

\begin{tabular}{|c|c|c|}
\hline Stage & Mean GA (Weeks) & Mean Birth Weight (Grams) \\
\hline Stage 1 & $31.8( \pm 2.52)$ & $1360( \pm 238)$ \\
\hline Stage 2 & $30( \pm 2)$ & $1216( \pm 288)$ \\
\hline Stage 3 & $31.5( \pm 2.12)$ & $1150( \pm 70.7)$ \\
\hline Stage 4 & 30 & 1200 \\
\hline Stage 5 & 33 & 1300 \\
\hline \multicolumn{2}{|c|}{ Table 3. Stage of ROP with Mean ( $\begin{array}{c}\text { SD) GA and Birth } \\
\text { Weight }\end{array}$} \\
\hline
\end{tabular}

\begin{tabular}{|c|c|c|}
\hline Risk Factor & ROP Cases & Percentage \\
\hline Respiratory distress & 13 & $81.25 \%$ \\
\hline Birth asphyxia & 2 & $12.5 \%$ \\
\hline Sepsis & 7 & $43.75 \%$ \\
\hline Neonatal jaundice & 6 & $37.5 \%$ \\
\hline \multicolumn{2}{|c|}{ Table 4. Distribution of Risk Factors among ROP Cases } \\
\hline
\end{tabular}


The table below shows the outcome of ROP in studied cases. Intervention with laser was necessary for the eight cases and patients showed improvement on follow-up. Of all sixteen cases that developed ROP, 8 cases required laser photocoagulation accounting for 50 percent of ROP cases and five percent of the total screened infants. The other eight cases regressed spontaneously without intervention.

\begin{tabular}{|c|c|c|}
\hline ROP Stage & Number & Outcome \\
\hline Stage 1 & 8 & $\begin{array}{c}\text { Spontaneous regression on follow-up } \\
\text { Needed laser and improved } \\
\text { (plus disease) }\end{array}$ \\
\hline Stage 2 & 3 & $\begin{array}{c}\text { Needed laser and improved } \\
\text { (plus disease) }\end{array}$ \\
\hline Stage 3 & 2 & Needed laser and improved \\
\hline Stage 4 & 1 & Needed laser and improved \\
\hline Stage 5 & 1 & Needed laser and improved \\
\hline \multicolumn{2}{|c|}{ Table 5. Outcome of ROP in Studied Cases } \\
\hline
\end{tabular}

\section{DISCUSSION}

The World Health Organisation Vision 2020 programme has identified ROP as an important cause of blindness.

The incidence of ROP in our study was an overall $10 \%$. Our results are very much similar to the incidence of developed countries of range less than 20\%.(14-16) The incidence in our study is similar to the Beijing study.(17) This value is very much less compared to other studies from our country, which ranges from $20 \%-47 \% .{ }^{(18-21)}$ This improvement is probably due to the increase in health care facilities, increased awareness, improved screening program for ROP and using higher cut-offs for birth weight and gestational age.

The risk factors reported in our study are respiratory distress in $81.25 \%$ cases of ROP followed by sepsis in $43.75 \%$. Other studies have also reported similar risk factors indicating that ROP is a disease of smallest and sickest babies. $(19,20)$

Hypoxia stimulates VEGF production, which induces neovascularisation at the border between vascularised and non-vascularised retina within the worst case is ending in retinal detachment. Down regulation of VEFG occurs due to hyperoxia. However, due to increased oxygen demand of fast developing eye, VEGF is upregulated which results in ROP.

Sepsis may act through cytokines and endotoxins or by oxidative burst in the neutrophils consequent to infection. In this study, we found that sepsis was also a major contributor with the development of ROP.(22,23) Its prevention and early control may reduce the incidence of ROP.

In our study, ROP was seen in male babies with predominance of $81.25 \%$, similar to the previous study by Darlow.(24)

Lower gestational age and low birth weight are the most important risk factors for the aetiology for ROP in our study and it is well recognised that the incidence and severity of ROP are inversely proportional to gestational age and birth weight.

In our study, the mean gestational age of any stage ROP was 31.2 weeks and average birth weight was 1290 grams (990 gms - 1600 gms) which are in consistent with the previous studies.(18,22)

Different countries follow different ROP screening guidelines. American Academy of Paediatrics recommended to screen all neonates with a birth weight $<1500$ gms or gestational age $\leq 28$ weeks, as well as selected children between 1500 and 2000 gms with an unstable course who are believed to be at high risk by their attending paediatricians.(25) National Neonatology Forum of India has recommended the guidelines for ROP screening of Indian babies. (26) According to it, all preterm neonates who are $<34$ weeks of gestation and/or $<1750$ gms birth weight should be screened for ROP.

In some studies, the inclusion criteria for patients was less gestational age and/or weight, while in some other studies either gestational age or only weight was considered as inclusion criteria. In order to include all ROP positive cases, we have included all babies with gestational age less than 36 weeks and/or birth weight less than 2000 gms as suggested by Jalali.(7) In our setup we have found ROP positive cases both above and below the gestational age of 32 weeks and birth weight of 1500 grams. As the inclusion criteria are different, it is not possible to compare results fully. Thus, the screening criteria should be less rigid than described in reports from long established centres.

Laser photocoagulation was found to be very effective in regressing ROP. In agreement with previous study, (1) we found that the eight cases that required laser intervention improved and ROP regressed with regular follow-up. Laser is now the preferred mode, since the most severe forms of the disease are more easily treated with laser than with cryotherapy.(27)

\section{CONCLUSION}

Screening for ROP aids in timely diagnosis and would facilitate early interventional strategies leading to prevention of ocular morbidity. The incidence of ROP is relatively less in our setup, probably due to monitored oxygen therapy and proper screening protocols. The reduced incidence could also be due to low survival of extreme premature babies and limited sample size. Thus, the screening criteria should be less rigid and different regions should make guidelines based on current and local data.

\section{ACKNOWLEDGEMENT}

Dr. Sai Kiranmayee (MS Ophthal), Consultant Vitreoretinal Surgeon, Pushpagiri Eye Institute, Secunderabad.

\section{REFERENCES}

[1] Coats DK, Miller AM, Hussein MAW, et al. Involution of retinopathy of prematurity after laser treatment: factors associated with development of retinal detachment. Am J Ophthalmol 2005;140(2):214-22.

[2] Azad R, Chandra P. Retinopathy of prematurity. J Indian Med Assoc 2005;103(7):370-2.

[3] Tasman W. Retinopathy of prematurity: do we still have a problem? The Charles L. Schepens lecture. Arch Ophthalmol 2011;129(8):1083-6.

[4] Maida JM, Mathers K, Alley CL. Pediatric ophthalmology in the developing world. Curr Opin Ophthalmol 2008;19(5):403-8.

[5] Gilbert C, Fielder A, Gordillo L, et al. Characteristics of infants with severe retinopathy of prematurity in countries with low, moderate and high levels of development: implications for screening programs. Pediatrics 2005;115(5):e518-25.

[6] 11_Retinopa.pdf 2017. http://www.sankaranethralaya.org/insight/PDF\%20 Files/July15/11_RETINOPA.pdf 
[7] Jalali S, Anand R, Kumar H, et al. Programme planning and screening strategy in retinopathy of prematurity. Indian J Ophthalmol 2003;51(1):89-99.

[8] Smith LEH. Pathogenesis of retinopathy of prematurity. Semin Neonatol 2003;8(6):469-73.

[9] Flynn JT. Acute proliferative retrolental fibroplasia: multivariate risk analysis. Trans Am Ophthalmol Soc 1983;81:549-91.

[10] Terry TL. Extreme prematurity and fibroblastic overgrowth of persistent vascular sheath behind each crystalline lens. Am J Ophthalmol 1942;25(2):203-4.

[11] Heath P. Pathology of the retinopathy of prematurity: retrolental fibroplasia. Am J Ophthalmol 1951;34(9):1249-59.

[12] International Committee for the Classification of Retinopathy of Prematurity. The International classification of retinopathy of prematurity revisited. Arch Ophthalmol 2005;123(7):991-9.

[13] Early Treatment for Retinopathy of Prematurity Cooperative Group. Revised indications for the treatment of retinopathy of prematurity: results of the early treatment for retinopathy of prematurity randomized trial. Arch Ophthalmol 2003;121(12):1684-94.

[14] Chiang MF, Arons RR, Flynn JT, et al. Incidence of retinopathy of prematurity from 1996 to 2000: analysis of a comprehensive New York state patient database. Ophthalmology 2004;111(7):1317-25.

[15] Tasman W, Patz A, McNamara JA, et al. Retinopathy of prematurity: the life of a lifetime disease. Am J Ophthalmol 2006;141(1):167-74.

[16] Chen J, Stahl A, Hellstrom A, et al. Current update on retinopathy of prematurity: screening and treatment. Curr Opin Pediatr 2011;23(2):173-8.

[17] Chen Y, Li XX, Yin H, et al. Risk factors for retinopathy of prematurity in six neonatal intensive care units in Beijing, China. Br J Ophthalmol 2008;92(3):326-30.

[18] A study of risk factors for retinopathy of prematurity in a medical college hospital in south india to evaluate.pdf 2017. http://jctmb.com/Vol2-Issue2-Apr-Jun2014/05.A\%20Study\%200f\%20Risk\%20Factors\%20 For\%20Retinopathy\%200f\%20Prematurity $\% 20$ In $\% 2$ 0A\%20Medical\%20College\%20Hospital\%20In\%20So uth\%20India\%20To\%20Evaluate.pdf
[19] Chaudhari S, Patwardhan V, Vaidya U, et al. Retinopathy of prematurity in a tertiary care center--incidence, risk factors and outcome. Indian Pediatr 2009;46(3):21924.

[20] Gupta VP, Dhaliwal U, Sharma R, et al. Retinopathy of prematurity--risk factors. Indian $\mathrm{J}$ Pediatr 2004;71(10):887-92.

[21] Charan R, Dogra MR, Gupta A, et al. The incidence of retinopathy of prematurity in a neonatal care unit. Indian J Ophthalmol 1995;43(3):123-6.

[22] Vinekar A, Dogra MR, Sangtam T, et al. Retinopathy of prematurity in Asian Indian babies weighing greater than 1,250 grams at birth: Ten year data from a tertiary care center in a developing country. Indian J Ophthalmol 2007;55(5):331-6.

[23] Shah VA, Yeo CL, Ling YLF, et al. Incidence, risk factors of retinopathy of prematurity among very low birth weight infants in Singapore. Ann Acad Med Singapore 2005;34(2):169-78.

[24] Darlow BA, Hutchinson JL, Henderson-Smart DJ, et al. Prenatal risk factors for severe retinopathy of prematurity among very preterm infants of the Australian and New Zealand Neonatal Network. Pediatrics 2005;115(4):990-6.

[25] Ophthalmology AAOPS on, Ophthalmology AAO, Strabismus AA for POA, Orthoptists AA of C. Screening examination of premature infants for retinopathy of prematurity. Pediatrics 2013;131(1):189-95.

[26] nnpd_report_2002-03.pdf 2017. http://www.newbornwhocc.org/pdf/nnpd_report_20 02-03.PDF

[27] Cole C, Fielder A, Schulenburg E. Management of retinopathy of prematurity. Curr Paediatr 2005;15(2):99-105. 